\title{
A Stickelberger theorem for $p$-adic Gauss sums
}

\author{
by \\ RÉGIS Blache (Pointe à Pitre)
}

0. Introduction. Character sums over finite fields have been widely studied since Gauss. During the last decade, coding theorists initiated the study of a new type of sums, over points with coordinates in a $p$-adic field. Let $\mathcal{O}_{m}^{(u)}$ be the ring of integers of $K_{m}=\mathbb{Q}_{p}\left(\zeta_{p^{m}-1}\right)$, the unramified extension of degree $m$ of the field of $p$-adic numbers $\mathbb{Q}_{p}$ obtained by adjoining the $\left(p^{m}-1\right)$ th roots of unity, and $\mathcal{T}_{m}=\mathcal{T}_{m}^{*} \cup\{0\}$ the Teichmüller of $\mathcal{O}_{m}^{(u)}$, where $\mathcal{T}_{m}^{*}$ is the multiplicative subgroup of elements of finite order in $\mathcal{O}_{m}^{(u) *}$. Many character sums over finite fields can be extended to this situation; the first studied was $\sum_{x \in \mathcal{T}_{m}} \widetilde{\psi}_{l, m}(f(x)), \widetilde{\psi}_{l, m}$ an additive character of order $p^{l}$ over $\mathcal{O}_{m}^{(u)}, f$ a polynomial in $\mathcal{O}_{m}^{(u)}[X]$, in order to give a generalization of the Weil-Carlitz-Uchiyama bound (cf. [6], and note that for $l=1$, we get the sums associated to a polynomial and an additive character over the finite field with $p^{m}$ elements, $\mathbb{F}_{p^{m}}$ ).

In this paper we are concerned with certain sums looking as Gauss sums; precisely, if $\widetilde{\psi}_{l, m}$ is as above, and $\chi$ is a multiplicative character of order dividing $p^{m}-1$, we define the $p$-adic Gauss sum of level $l$ associated with the characters $\widetilde{\psi}_{l, m}$ and $\chi$ as

$$
G_{\mathcal{T}_{m}}\left(\widetilde{\psi}_{l, m}, \chi\right)=\sum_{x \in \mathcal{T}_{m}^{*}} \widetilde{\psi}_{l, m}(x) \chi(x) .
$$

These sums have already been studied in [11], where a bound is given, and in [8], which has been our starting point.

Here we do not give a bound; we focus on the $p$-adic valuation of these character sums. Our aim is to generalize the following theorem of Stickelberger on classical Gauss sums to this situation. We first recall some notations: let $\widetilde{\psi}_{1, m}$ be an additive character of order $p$ on $\mathcal{O}_{m}^{(u)}$, and $\chi$ a

2000 Mathematics Subject Classification: 11L05, 11L07, 11T24.

Key words and phrases: Gauss sums, Stickelberger theorem, splitting functions, Witt vectors. 
multiplicative character of order exactly $p^{m}-1$, both taking values in $\mathbb{C}_{p}$, the (algebraically closed) completion of an algebraic closure of $\mathbb{Q}_{p}$. If we set $\gamma=\widetilde{\psi}_{1, m}(1)-1$, then $\gamma$ is a generator of the maximal ideal of the ring of integers of $\Omega_{1}$, the ramified extension of degree $p-1$ of $\mathbb{Q}_{p}$ obtained by adjoining the $p$ th roots of unity. Let $K_{1, m}$ be the compositum of $\Omega_{1}$ and $K_{m}$. In this situation, the Gauss sums of level 1 are exactly the classical Gauss sums over finite fields (cf. [2]), and we have:

TheOREM (Stickelberger, [7]). In $\mathcal{O}_{1, m}$, the ring of integers of $K_{1, m}$, for any $0 \leq a \leq p^{m}-2$, we have

$$
G_{\mathcal{T}_{m}}\left(\chi^{-a}, \widetilde{\psi}_{1, m}\right) \equiv-\frac{\gamma^{s(a)}}{p(a)}\left[\gamma^{s(a)+1}\right]
$$

where if $a=a_{0}+p a_{1}+\cdots+p^{m-1} a_{m-1}$ is the $p$-adic expansion of the integer $a$, we define $s(a):=a_{0}+a_{1}+\cdots+a_{m-1}$ and $p(a):=a_{0} ! \cdots a_{m-1}$ !.

We use ideas close to Dwork's in his proof of the rationality of the zeta functions of varieties over finite fields (cf. [4]); our generalization of this theorem relies on an improvement of his splitting functions. Roughly speaking, a splitting function, in the sense of Dwork, is an analytic representation of an additive character of order $p$ with values in $\mathbb{C}_{p}^{*}$; it is a power series $\Theta$ with coefficients in $\Omega_{1}$, converging in the closed disk of center 0 and radius 1 , and such that for any $t \in \mathcal{T}:=\mathcal{T}_{1}, \Theta(t)=\psi_{1}(\bar{t})$, where $\bar{t}$ is the image of $t$ in the finite field with $p$ elements $\mathbb{F}_{p} \simeq \mathbb{Z}_{p} / p \mathbb{Z}_{p}$, and $\psi_{1}$ is a nontrivial additive character of $\mathbb{F}_{p}$. Let us give an example of such a function. Consider the series

$$
G(X)=\sum_{i \geq 0} \frac{X^{p^{i}}}{p^{i}} \in \mathbb{Q}_{p}[[X]] .
$$

It has $p-1$ zeroes of valuation $(p-1)^{-1}$, and if $\gamma_{1}$ is one of them, then the series $\theta_{1}(X):=\exp \left(G\left(\gamma_{1} X\right)\right)$ converges in the closed unit disk, and is a splitting function.

The main problem here is to extend this concept to additive characters of order $p^{l}$. We shall define splitting functions of level $l$, in order to give an analytic representation of an additive character $\widetilde{\psi}_{l}$ of $\mathbb{Z}_{p}$ of order $p^{l}$. The first point is to define functions representing the $p^{l}$ th roots of unity; in order to do this we follow closely the ideas of Dwork, replacing $\gamma_{1}$ as above by $\gamma_{l}$, a zero of $G$ of valuation $\left(p^{l-1}(p-1)\right)^{-1}$, and defining $\theta_{l}(X):=\exp \left(G\left(\gamma_{l} X\right)\right)$. Then we use the Witt vectors representation of the rings $\mathbb{Z}_{p} / p^{l} \mathbb{Z}_{p}$ and $\mathcal{O}_{m}^{(u)} / p^{l} \mathcal{O}_{m}^{(u)}$. Since $\mathbb{Z}_{p} / p^{l} \mathbb{Z}_{p} \simeq \mathbb{Z} / p^{l} \mathbb{Z} \simeq W_{l}\left(\mathbb{F}_{p}\right)$, the ring of Witt vectors of length $l$ with coefficients in $\mathbb{F}_{p}$, we shall require a splitting function $\Theta_{l}$ to satisfy, for any $\left(t_{0}, \ldots, t_{l-1}\right)$ in $\mathcal{T}^{l}$, the condition that $\Theta_{l}\left(t_{0}, \ldots, t_{l-1}\right)=\psi_{l}\left(\bar{t}_{0}, \ldots, \bar{t}_{l-1}\right)$, where $\psi_{l}$ is a character of $W_{l}\left(\mathbb{F}_{p}\right)$ via the above isomorphisms. Once this 
has been done, the generalization of Stickelberger's theorem is an easy consequence of the description of the coefficients of the splitting function.

The paper is organized as follows: we begin Section 1 by recalling some useful results on $p^{l}$ th roots of unity in $\mathbb{C}_{p}$. Then we use the Artin-Hasse exponential and the roots of Artin-Hasse power series to construct power series whose values at the elements of $\mathcal{T}$ are $p^{l}$ th roots of unity; note that this construction is very close to Dwork's construction of his splitting functions (cf. [4]). In Section 2, after recalling some definitions about rings of Witt vectors, we define splitting functions of level $l$, and we give an example of such a function relying on the preceding construction; this is Theorem 2.3. The key lemma in its proof is Lemma 2.4. It gives a link between the shape of Artin-Hasse power series and certain polynomials arising from the addition of Witt vectors, and allows us to show that our function represents an additive character. Section 3 is devoted to the proof of Stickelberger's theorem.

Notations. In this paper, $\mathbb{Q}_{p}$ is the field of $p$-adic numbers, $\mathbb{Z}_{p}$ the ring of $p$-adic integers; let $\mathcal{T}:=\left\{x \in \mathbb{Z}_{p} ; x^{p}=x\right\} \subset \mathbb{Z}_{p}$ be the Teichmüller of $\mathbb{Z}_{p}$. Note that $\mathcal{T}=\mathcal{T}^{\times} \cup\{0\}$, where $\mathcal{T}^{\times}=\left\{x \in \mathbb{Z}_{p} ; x^{p-1}=1\right\}$ is the subgroup of elements of finite order in $\mathbb{Z}_{p}^{\times}$. Let also $k:=\mathbb{F}_{p}$ be the residue field of $\mathbb{Z}_{p}$. Note that $\mathcal{T}$ is the image of a lifting of $k$ to $\mathbb{Z}_{p}$, called the Teichmüller lifting.

We denote by $\mathbb{C}_{p}$ a completion of the algebraic closure of $\mathbb{Q}_{p}$, and by $v_{p}$ the $p$-adic valuation on $\mathbb{C}_{p}$, normalized such that $v_{p}(p)=1$. Let $K_{m}$ be the extension of $\mathbb{Q}_{p}$ generated by the $\left(p^{m}-1\right)$ th roots of unity; it is well known that $K_{m}$ is an unramified extension of degree $m$ of $\mathbb{Q}_{p}$. We denote by $\mathcal{O}_{m}^{(u)}$ its valuation ring, and by $\mathcal{T}_{m}$ its Teichmüller, $\mathcal{T}_{m}:=$ $\left\{x \in \mathcal{O}_{m}^{(u)} ; x^{p^{m}}=x\right\}$; once again, $\mathcal{T}_{m}=\mathcal{T}_{m}^{\times} \cup\{0\}$, where $\mathcal{T}_{m}^{\times}=\left\{x \in \mathbb{Z}_{p} ;\right.$ $\left.x^{p^{m}-1}=1\right\}$ is the subgroup of elements of finite order in $\mathcal{O}_{m}^{(u) \times}$, and $\mathcal{T}_{m}$ is the image of the Teichmüller lifting, a lifting of $k_{m}:=\mathbb{F}_{p^{m}}$ to $\mathcal{O}_{m}^{(u)}$. Recall that any element $x \in K_{m}$ can be written uniquely as

$$
x=p^{-r} t_{-r}+\cdots+t_{0}+p t_{1}+\cdots
$$

for some $t_{-r}, \ldots, t_{0}, t_{1}, \ldots$ in $\mathcal{T}_{m}$.

The extension $K_{m} / \mathbb{Q}_{p}$ is Galois, with cyclic Galois group of order $m$, generated by the Frobenius $F$, whose action on $x=p^{-r} t_{-r}+\cdots+t_{0}+p t_{1}+\cdots$ is

$$
F(x)=p^{-r} t_{-r}^{p}+\cdots+t_{0}^{p}+p t_{1}^{p}+\cdots .
$$

We can also define a trace from $K_{m}$ to $\mathbb{Q}_{p}$ by $\operatorname{Tr}(x)=x+F(x)+\cdots+$ $F^{m-1}(x)$.

Let $l \geq 1$ be an integer. We let $R:=R_{1}$ be the $\operatorname{ring} \mathbb{Z} / p^{l} \mathbb{Z}=\mathbb{Z}_{p} / p^{l} \mathbb{Z}_{p}$, and $R_{m}=\mathcal{O}_{m}^{(u)} / p^{l} \mathcal{O}_{m}^{(u)}$. Note that the action of Frobenius passes to the 
quotient; in this way, we can consider the ring $R_{m}$ as the unramified cyclic extension of $R$ of degree $m$, adopting the terminology in [9].

1. Analytic representation of $p^{l}$ th roots of unity. We begin this section by giving properties of $p^{l}$ th roots of unity, and the extensions of $\mathbb{Q}_{p}$ they generate, in Subsection 1.1. Then, in Subsection 1.2, we consider series whose values at elements of $\mathcal{T}^{\times}$are such roots. Dwork has already shown that the series

$$
G(X)=\sum_{i \geq 0} \frac{X^{p^{i}}}{p^{i}} \in \mathbb{Q}_{p}[[X]]
$$

has $p-1$ zeroes of valuation $(p-1)^{-1}$, and that given one of these zeroes, say $\gamma_{1}$, the values of the series $\theta_{1}(X):=\exp \left(G\left(\gamma_{1} X\right)\right)$ at elements of $\mathcal{T}^{\times}$ are $p$ th roots of unity. Here we generalize this remark to $p^{l}$ th roots of unity, using the roots of $G$ of valuation $\left(p^{l-1}(p-1)\right)^{-1}$.

1.1. The fields generated by $p^{l}$ th roots of unity. Let $\Omega_{l}:=\mathbb{Q}_{p}\left(\zeta_{p^{l}}\right)$ denote the extension of $\mathbb{Q}_{p}$ obtained by adjoining a primitive $p^{l}$ th root of unity $\zeta_{p^{l}}$, and $\mathcal{O}_{l}^{(r)}$ its ring of integers.

Lemma 1.1. The field $\Omega_{l}$ is a totally ramified extension of $\mathbb{Q}_{p}$, of degree $p^{l}-p^{l-1} ;$ moreover, we have

$$
v_{p}\left(\zeta_{p^{l}}-1\right)=\left(p^{l}-p^{l-1}\right)^{-1},
$$

that is, $\zeta_{p^{l}}-1$ is a generator of the maximal ideal $m_{l}^{(r)}$ of the local ring $\mathcal{O}_{l}^{(r)}$.

Proof. Clearly a primitive $p^{l}$ th root of unity is a root of the polynomial

$$
\left(X^{p^{l}}-1\right) /\left(X^{p^{l-1}}-1\right)=X^{(p-1) p^{l-1}}+\cdots+X^{p^{l-1}}+1 .
$$

Thus $\zeta_{p^{l}}-1$ is a root of $P(X):=(X+1)^{(p-1) p^{l-1}}+\cdots+(X+1)^{p^{l-1}}+1$. Looking modulo $p$, we get

$$
\begin{aligned}
P(X) & \equiv\left(X^{p^{l-1}}+1\right)^{p-1}+\cdots+\left(X^{p^{l-1}}+1\right)+1[p] \\
& \equiv \sum_{k=1}^{p}\left(\sum_{i=1}^{k} C_{p-i}^{k-i}\right) X^{(p-k) p^{l-1}}[p] .
\end{aligned}
$$

Now since $C_{p-i}^{k-i} \equiv(-1)^{k-i} C_{k-1}^{i-1}[p]$, the coefficient of degree $k$ is zero modulo $p$ for $k \geq 2$; we get $P(X) \equiv X^{(p-1) p^{l-1}}[p]$. Since the constant term of $P$ is $p$, it is an Eisenstein polynomial, thus irreducible, and all its roots are of valuation $\left(p^{l-1}(p-1)\right)^{-1}$, generating the maximal ideal of $\mathcal{O}_{l}^{(r)}$.

The following corollary will be useful: 
Corollary 1.2. Let $\zeta$ and $\zeta^{\prime}$ be two $p^{l}$ th roots of unity in $\mathbb{C}_{p}$. If $v_{p}\left(\zeta-\zeta^{\prime}\right)$ $>1$, then $\zeta=\zeta^{\prime}$.

Proof. Since $\zeta-\zeta^{\prime}=\zeta^{\prime}\left(\zeta\left(\zeta^{\prime}\right)^{-1}-1\right)$, we have $v_{p}\left(\zeta\left(\zeta^{\prime}\right)^{-1}-1\right)>1$, and $\zeta\left(\zeta^{\prime}\right)^{-1}$ is a $p^{k}$ th root of unity for some $0 \leq k \leq l$. Thus from Lemma 1.1, we must have $k=0$, that is, $\zeta=\zeta^{\prime}$.

1.2. Analytic representation of $p^{l}$ th roots of unity. Let us begin by a quick overview of the theory of Newton polygons (cf. [1, Chapter 4.3], [5, Chapter IV]). If $f(X):=\sum_{n>0} a_{n} X^{n}$ is a polynomial or a power series in $\mathbb{C}_{p}[[X]]$, its Newton polygon $\mathrm{NP}(f)$ is the convex hull of the set of points with coordinates $\left(n, v_{p}\left(a_{n}\right)\right), n \geq 0$. Then we have the following remarkable result (cf. [1, Theorem 4.4.4], [5, p. 106, Corollary to Theorem 14]): if NP(f) has an edge of (horizontal) length $l$ and slope $s$, then $f$ has exactly $l$ zeroes of valuation $-s$ in $\mathbb{C}_{p}$. Applying this result to $G$, we see that for any $k \geq 1$, $G(X)$ has $p^{k}-p^{k-1}$ zeroes of valuation $\left(p^{k}-p^{k-1}\right)^{-1}$ in $\mathbb{C}_{p}$.

LEMMA 1.3. Let $\gamma_{l}$ be a zero of $G$ with $v_{p}\left(\gamma_{l}\right)=\left(p^{l}-p^{l-1}\right)^{-1}, l \geq 2$. Then there is a unique root $\gamma_{l-1}$ of $G$ with valuation $\left(p^{l-1}-p^{l-2}\right)^{-1}$ such that $\gamma_{l-1} \equiv \gamma_{l}^{p}\left[p \gamma_{l}\right]$.

Proof. We will consider the Newton polygon of $G_{l}(X):=G\left(X+\gamma_{l}^{p}\right)$. Set $G_{l}(X):=\sum_{k \geq 0} f_{k} X^{k}$. For $k \geq 1$, a rapid calculation gives

$$
f_{k}=\sum_{i \geq\left\lceil\log _{p}(k)\right\rceil} \frac{C_{p^{i}}^{k}}{p^{i}} \gamma_{l}^{p\left(p^{i}-k\right)}
$$

Since $v_{p}\left(C_{p^{i}}^{k}\right)=i-v_{p}(k)$, we get $v_{p}\left(f_{k}\right) \geq-v_{p}(k)$. Moreover, if $k=p^{j}$, $j \geq 0$, the term with minimal valuation in $f_{k}$ is the term $i=j$, which is $p^{-j}$; we get $v_{p}\left(f_{p^{j}}\right)=-j$. Finally, the constant term is

$$
\sum_{k \geq 1} \frac{\gamma_{l}^{p^{k}}}{p^{k-1}}=p \sum_{k \geq 1} \frac{\gamma_{l}^{p^{k}}}{p^{k}}=p\left(G\left(\gamma_{l}\right)-\gamma_{l}\right)=-p \gamma_{l}
$$

which is of valuation $1+\left(p^{l}-p^{l-1}\right)^{-1}$. Thus the Newton polygon of $G_{l}$ has vertices $\left(0,1+\left(p^{l}-p^{l-1}\right)^{-1}\right)$ and $\left(p^{k},-k\right), k \geq 0$. It has an edge of length 1 with slope $-1-\left(p^{l}-p^{l-1}\right)^{-1}$, that is, $G_{l}$ has a unique zero of valuation $1+\left(p^{l}-p^{l-1}\right)^{-1}$, say $\alpha_{l}$, and all other zeroes are of valuation less than or equal to $(p-1)^{-1}$. Finally, $G$ has a unique zero $\gamma_{l-1}:=\gamma_{l}^{p}+\alpha_{l}$ such that $\gamma_{l-1} \equiv \gamma_{l}^{p}\left[p \gamma_{l}\right]$. Any other zero $\gamma$ satisfies $v_{p}\left(\gamma-\gamma_{l}^{p}\right) \leq(p-1)^{-1}$.

From the above lemma, we fix once and for all a "compatible" sequence $\left(\gamma_{k}\right)_{0 \leq k \leq l}$ in $\mathbb{C}_{p}$, that is, $\gamma_{l}$ is a fixed root of $G$ of valuation $\left(p^{l}-p^{l-1}\right)^{-1}$, for $1 \leq \bar{k} \leq l-1, \gamma_{k}$ is a zero of $G(X)$ of valuation $\left(p^{k}-p^{k-1}\right)^{-1}$ such that $\gamma_{k} \equiv \gamma_{k+1}^{p}\left[p \gamma_{k+1}\right]$, and $\gamma_{0}:=0$, the trivial zero of $G$. 
Definition 1.4. Let us define the Artin-Hasse exponential as $\mathrm{AH}(X)$ $:=\exp (G(X))$, and $\theta_{k}(X):=\mathrm{AH}\left(\gamma_{k} X\right), 0 \leq k \leq l$.

First recall that from Dwork's lemma (cf. [5, IV.2, Lemma 3]), the series $\operatorname{AH}(X)$ is in $1+X \mathbb{Z}_{p}[[X]]$. Let us deduce some properties of $\theta_{k}$ from this result.

Proposition 1.5. Let $1 \leq k \leq l$. If the development of $\theta_{k}$ in power series in $\mathbb{C}_{p}[[X]]$ is

then its coefficients satisfy

$$
\theta_{k}(X)=\sum_{n \geq 0} \lambda_{n, k} X^{n}
$$

$$
v_{p}\left(\lambda_{n, k}\right) \geq \frac{n}{p^{k}-p^{k-1}}, \quad n \geq 0, \quad \lambda_{n, k}=\frac{\gamma_{k}^{n}}{n !}, \quad 0 \leq n \leq p-1 .
$$

In particular the function $\theta_{k}$ converges in the open disk with center 0 and radius $p^{1 /\left(p^{k}-p^{k-1}\right)}$.

Proof. Write $\mathrm{AH}(X)=\sum_{i>0} e_{i} X^{i}$. From Dwork's lemma, we know that $e_{0}=1$ and $e_{i} \in \mathbb{Z}_{p}$, that is, $v_{p}\left(e_{i}\right) \geq 0$. Now from the definition of $\theta_{k}$, we have $\lambda_{n, k}=e_{n} \gamma_{k}^{n}$, and $v_{p}\left(\lambda_{n, k}\right) \geq v_{p}\left(\gamma_{k}^{n}\right)=n /\left(p^{k}-p^{k-1}\right)$. This shows the first assertion.

Moreover, the first $p$ terms are those of the development of $\exp \left(\gamma_{k} X\right)$ in power series; we get $\lambda_{n, k}=\gamma_{k}^{n} / n$ ! for $0 \leq n \leq p-1$.

Now we show that from the compatible system $\left(\gamma_{k}\right)_{0 \leq k \leq l}$ and the functions $\theta_{k}$, we can define a compatible system of $p^{l}$ th roots of unity $\left(\zeta_{p^{k}}\right)_{0 \leq k \leq l}$ in the following sense:

Definition 1.6. For all $0 \leq k \leq l$, let $\zeta_{p^{k}}$ be a $p^{k}$ th root of unity in $\mathbb{C}_{p}$. We say that the family $\left(\zeta_{p^{k}}\right)_{0 \leq k \leq l}$ forms a compatible system of $p^{k}$ th roots of unity in $\mathbb{C}_{p}$ if the following holds:

(i) $\zeta_{p^{k}}$ is a primitive $p^{k}$ th root of unity,

(ii) $\zeta_{p^{k}}^{p}=\zeta_{p^{k-1}}$ for $1 \leq k \leq l$.

Proposition 1.7. The family $\left(\theta_{k}(1)\right)_{0 \leq k \leq l}$ forms a compatible system of $p^{l}$ th roots of unity.

Proof. Clearly, since $\theta_{0}(X)=1$, we have $\theta_{0}(1)=1$. Now we show that $\theta_{k}(1)$ is a primitive $p^{k}$ th root of unity for $1 \leq k \leq l$. From the description of $\theta_{k}$ in Proposition 1.5, we have

$$
\theta_{k}(1)=\sum_{n \geq 0} \lambda_{n, k}=1+\gamma_{k}+\cdots
$$

(this series converges by Proposition 1.5); this gives in particular

$$
v_{p}\left(\theta_{k}(1)-1\right)=\left(p^{k-1}(p-1)\right)^{-1} .
$$

Thus from Lemma 1.1 it just remains to show that $\theta_{k}(1)^{p^{k}}=1$. 
From the equality of formal power series $\exp (X)^{p^{k}}=\exp \left(p^{k} X\right)$, we get

$$
\mathrm{AH}(X)^{p^{k}}=\exp \left(p^{k} G(X)\right) .
$$

Let $x$ be an element of $\mathbb{C}_{p}$ with $v_{p}(x) \geq\left(p^{k}-p^{k-1}\right)^{-1}$; then

$$
v_{p}\left(p^{k} \frac{x^{p^{i}}}{p^{i}}\right) \geq \frac{p^{i-k+1}}{p-1}-i+k ;
$$

this quantity is minimal for $i=k-1, k$, and equals then $1+(p-1)^{-1}$; thus $v_{p}\left(p^{k} G(x)\right) \geq 1+(p-1)^{-1}$, and $p^{k} G(x)$ is in the convergence disk of the exponential. The above equality of formal power series gives $\mathrm{AH}(x)^{p^{k}}=$ $\exp \left(p^{k} G(x)\right)$, and we get

$$
\theta_{k}(1)^{p^{k}}=\operatorname{AH}\left(\gamma_{k}\right)^{p^{k}}=\exp \left(p^{k} G\left(\gamma_{k}\right)\right)=\exp (0)=1 .
$$

Now we show that $\theta_{k}(1)^{p}=\theta_{k-1}(1)$ for $1 \leq k \leq l$. We have

$$
\theta_{k}(1)^{p}=\left(\sum_{n \geq 0} \lambda_{n, k}\right)^{p} \equiv \sum_{n \geq 0} \lambda_{n, k}^{p}\left[p \gamma_{k}\right]
$$

since $\lambda_{n, k} \equiv 0\left[\gamma_{k}\right]$ for $n \geq 1$. Moreover we have $\lambda_{0, k}=\lambda_{0, k-1}=1$, and $\lambda_{n, k}^{p}=e_{n}^{p}\left(\gamma_{k}^{p}\right)^{n} \equiv e_{n} \gamma_{k-1}^{n}=\lambda_{n, k-1}\left[p \gamma_{k}\right]$ for $n \geq 1$ since $\gamma_{k}^{p} \equiv \gamma_{k-1}\left[p \gamma_{k}\right]$ by Lemma 1.3 and the $e_{n}$ are in $\mathbb{Z}_{p}$. Finally, we get

$$
\begin{aligned}
\theta_{k-1}(1)=\sum_{n \geq 0} e_{n} \gamma_{k-1}^{n} & \equiv \sum_{n \geq 0} e_{n} \gamma_{k}^{n p}\left[p \gamma_{k}\right] \\
& \equiv \sum_{n \geq 0} \lambda_{n, k}^{p}\left[p \gamma_{k}\right] \equiv \theta_{k}(1)^{p}\left[p \gamma_{k}\right] .
\end{aligned}
$$

Since both sides are $p^{k}$ th roots of unity, the result now follows from Corollary 1.2 .

In the following, we set $\zeta_{p^{k}}:=\theta_{k}(1)$ for all $0 \leq k \leq l$.

REMARK 1.8. (i) If $t \in \mathcal{T}^{*}$, then $t \gamma_{k}$ is also a root of $G$ of valuation $\left(p^{k-1}(p-1)\right)^{-1}$, and the proof of Proposition 1.7 shows that $\theta_{k}(t)=\mathrm{AH}\left(t \gamma_{k}\right)$ is also a primitive $p^{k}$ th root of unity.

(ii) We also see that the fields $\Omega_{k}$ and $\mathbb{Q}_{p}\left(\gamma_{k}\right)$ are equal (they are both totally ramified extensions of degree $p^{k}-p^{k-1}$ of $\mathbb{Q}_{p}$, and Proposition 1.7 implies that $\Omega_{k} \subset \mathbb{Q}_{p}\left(\gamma_{k}\right)$ ); thus $\gamma_{k}$ and $\zeta_{p^{k}}-1$ are both generators of the maximal ideal of $\Omega_{k}$ such that $\zeta_{p^{k}}-1 \equiv \gamma_{k}\left[\gamma_{k}^{2}\right]$.

2. The splitting functions of level $l$. When proving the rationality of zeta functions of varieties over finite fields, one of the main ideas of Dwork was that of a splitting function, i.e. power series over $\mathbb{C}_{p}$ "representing" additive characters over finite fields. The aim of this section is to extend this concept to the rings $R$ and $R_{m}$. In fact, via Witt vectors, any element 
of these rings can be represented as an $l$-tuple of elements of their residue fields $k$ and $k_{m}$; our strategy is to use coordinatewise Teichmüller lifting of these vectors and the results of Section 1 (in particular Remark 1.8) to construct power series of $l$ variables representing characters of $R$ and $R_{m}$.

In 2.1, we recall some facts about rings of Witt vectors, then in 2.2 , we give the link between the rings $R$ and $R_{m}$ and rings of Witt vectors with coordinates in the fields $k$ and $k_{m}$. Finally, in 2.3, we give a precise definition of the splitting functions of level $l$ and give an example of such a function.

2.1. Some background on rings of Witt vectors. We recall here the definitions and some properties of rings of Witt vectors; the reader can find more details in [10] or [3].

The Witt polynomials $\Phi_{0}, \ldots, \Phi_{n}, \ldots$ are defined in $\mathbb{Z}\left[\left(X_{i}\right)_{i \in \mathbb{N}}\right]$ by:

$$
\Phi_{0}\left(X_{0}\right)=X_{0}, \ldots, \Phi_{n}\left(X_{0}, \ldots, X_{n}\right)=X_{0}^{p^{n}}+p X_{1}^{p^{n-1}}+\cdots+p^{n} X_{n}, \ldots
$$

There exists two families of elements $\left(S_{i}\right)_{i \in \mathbb{N}},\left(P_{i}\right)_{i \in \mathbb{N}}$ in $\mathbb{Z}\left[\left(X_{i}, Y_{i}\right)_{i \in \mathbb{N}}\right]$ such that, for all $n \geq 0$,

$$
\begin{aligned}
\Phi_{n}\left(X_{0}, \ldots, X_{n}\right)+\Phi_{n}\left(Y_{0}, \ldots, Y_{n}\right) & =\Phi_{n}\left(S_{0}, \ldots, S_{n}\right), \\
\Phi_{n}\left(X_{0}, \ldots, X_{n}\right) \Phi_{n}\left(Y_{0}, \ldots, Y_{n}\right) & =\Phi_{n}\left(P_{0}, \ldots, P_{n}\right) .
\end{aligned}
$$

Note that if we assign the weight $p^{j}$ to the variables $X_{j}, Y_{j}$, then $S_{i}$ is isobarous of weight $p^{i}$, and $P_{i}$ is isobarous of weight $2 p^{i}$.

We are now ready to define rings of Witt vectors.

Definition 2.1. Let $A$ be a (commutative) ring. The ring of Witt vectors of length $l$, with coefficients in $A, W_{l}(A)$, is the set $A^{l}$, with addition $\oplus$ and multiplication $\otimes$ defined by

$$
\begin{aligned}
& \left(a_{0}, \ldots, a_{l-1}\right) \oplus\left(b_{0}, \ldots, b_{l-1}\right):=\left(S_{0}\left(a_{0}, b_{0}\right), \ldots, S_{l-1}\left(a_{0}, \ldots, b_{l-1}\right)\right), \\
& \left(a_{0}, \ldots, a_{l-1}\right) \otimes\left(b_{0}, \ldots, b_{l-1}\right):=\left(P_{0}\left(a_{0}, b_{0}\right), \ldots, P_{l-1}\left(a_{0}, \ldots, b_{l-1}\right)\right) .
\end{aligned}
$$

Note that for $0 \leq i \leq l-1$, the map $\Phi_{i}$ from $W_{l}(A)$ to $A$ is a ring homomorphism; the image of a vector in $W_{l}(A)$ by $\Phi_{i}$ is often called its $i t h$ ghost component.

As usual,

$$
V: W_{l}(A) \rightarrow W_{l}(A), \quad V\left(a_{0}, \ldots, a_{l-1}\right)=\left(0, a_{0}, \ldots, a_{l-2}\right),
$$

denotes the Verschiebung; it is a group endomorphism (for the additive structure of $\left.W_{l}(A)\right)$, but not a ring endomorphism. Note that for any vector $\left(a_{0}, \ldots, a_{l-1}\right)$ in $W_{l}(A)$, we have the equality of Witt vectors

$$
\left(a_{0}, \ldots, a_{l-1}\right)=\bigoplus_{i=0}^{l-1} V^{i}\left(a_{i}, 0, \ldots, 0\right) .
$$

Finally, if $\phi: A \rightarrow B$ is a ring homomorphism, then $W_{l}(\phi)$, the map from $W_{l}(A)$ to $W_{l}(B)$ sending $\left(a_{0}, \ldots, a_{l-1}\right)$ to $\left(\phi\left(a_{0}\right), \ldots, \phi\left(a_{l-1}\right)\right)$, is again a 
ring homomorphism. In particular, if $A$ is a ring of characteristic $p$, the Frobenius of $A$, sending an element to its $p$ th power, induces a homomorphism of $W_{l}(A)$, sending $\left(a_{0}, \ldots, a_{l-1}\right)$ to $\left(a_{0}^{p}, \ldots, a_{l-1}^{p}\right)$. We shall denote this morphism by $F$, and call it the Frobenius of $W_{l}(A)$.

2.2. The ring $\mathbb{Z} / p^{l} \mathbb{Z}$ and its unramified extensions. In this section, we recall results about the ring $\mathbb{Z} / p^{l} \mathbb{Z}$ and its unramified extensions; the proofs and more details can be found in [9]. The image of $\mathcal{T}_{m}$ under reduction modulo $p^{l}$ is a subset of $R_{m}$, which is the image of a section $\tau$ of reduction modulo $p$ from $R_{m}$ to $k_{m}$; moreover, every element $b$ in $R_{m}$ can be written in a unique way as $b_{0}+\cdots+p^{l-1} b_{l-1}$, with $b_{0}, \ldots, b_{l-1}$ in the image of $\mathcal{T}_{m}$.

The Galois group of the extension $R_{m} / R$ is cyclic of order $m$, generated by the Frobenius $F$ sending $b=b_{0}+\cdots+p^{l-1} b_{l-1}$ to $F(b)=b_{0}^{p}+\cdots+p^{l-1} b_{l-1}^{p}$. We also define a trace from $R_{m}$ to $R$ by

$$
\operatorname{Tr}_{R_{m} / R}(b)=b+F(b)+\cdots+F^{m-1}(b) .
$$

With the help of the trace, we can describe the additive characters of the rings $R_{m}, m \geq 1$. Let $\psi_{l}$ be a primitive additive character of $R$, i.e. sending the class of 1 to a primitive $p^{l}$ th root of unity; then every character of $R_{m}$ can be written as $y \mapsto \psi_{l}\left(\operatorname{Tr}_{R_{m} / R}(x y)\right)$ for some $x$ in $R_{m}$.

For any $m \geq 1$, we fix an isomorphism $w_{m}: W_{l}\left(k_{m}\right) \rightarrow R_{m}$ defined by:

$$
w_{m}\left(a_{0}, \ldots, a_{l-1}\right)=\tau\left(a_{0}\right)+p F^{-1} \tau\left(a_{1}\right)+\cdots+p^{l-1} F^{-(l-1)} \tau\left(a_{l-1}\right) .
$$

Note that via $w_{m}$, the Frobenius $F$ on $R_{m}$ corresponds to the Frobenius $F$ of $W_{l}\left(k_{m}\right)$ defined in 2.1. Moreover the trace from $R_{m}$ to $R$ corresponds to the trace from $W_{l}\left(k_{m}\right)$ to $W_{l}(k)$ defined by

$$
\operatorname{Tr}_{W_{l}\left(k_{m}\right) / W_{l}(k)}\left(a_{0}, \ldots, a_{l-1}\right)=\bigoplus_{i=0}^{m-1} F^{i}\left(a_{0}, \ldots, a_{l-1}\right)=\bigoplus_{i=0}^{m-1}\left(a_{0}^{p^{i}}, \ldots, a_{l-1}^{p^{i}}\right) .
$$

2.3. The splitting functions. Recall the concept of a splitting function, as introduced by Dwork (cf. [4]). A splitting function $\Theta$ is a power series in one variable over $\Omega_{1}$ that converges in a disk of radius strictly greater than 1 and has the following two properties:

(i) For $x$ in $k$, denote by $\widetilde{x} \in \mathcal{T}$ its Teichmüller representative, the unique element of $\mathcal{T}$ that reduces to $x$ modulo $p$. Then the function $x \mapsto \Theta(\widetilde{x})$ is a nontrivial additive character $\psi$ of $k$, with values in $\Omega_{1}$.

(ii) For each $m \geq 1$, the additive character $\psi_{m}$ of $k_{m}$ obtained by composing $\psi$ with the trace from $k_{m}$ to $k$ can be represented as follows: for $x$ in $k_{m}$, denote by $\widetilde{x} \in \mathcal{T}_{m}$ its Teichmüller representative; then we have

$$
\psi_{m}(x)=\prod_{i=0}^{m-1} \Theta\left(\widetilde{x}^{p^{i}}\right)
$$


We shall generalize this concept in order to represent additive characters of order $p^{l}$, i.e. additive characters of the rings $R_{m}$. We first need to give an equivalent of the Teichmüller lifting. For this we will use Witt vectors. From the isomorphisms $w_{m}$, we can define an equivalent of the Teichmüller representative and generalize the notion of splitting function.

Let $x \in R_{m}$ whose image under $w_{m}^{-1}$ is $\left(x_{0}, \ldots, x_{l-1}\right)$. We define the lift of $x$ as $\widehat{x}:=\left(\widetilde{x}_{0}, \ldots, \widetilde{x}_{l-1}\right)$, the element of $\mathcal{T}_{m}^{l} \subset \mathbb{C}_{p}^{l}$ obtained by replacing each component of the Witt vector corresponding to $x$ by its Teichmüller representative. We also define, for any $i \geq 0$,

$$
F^{i} \widehat{x}=\left(\widetilde{x}_{0}^{p^{i}}, \ldots, \widetilde{x}_{l-1}^{p^{i}}\right) ;
$$

note that it is the lift of $F^{i} x$.

We are now ready to define the splitting functions of level $l$ :

Definition 2.2. A splitting function of level $l, \Theta_{l}$, is a power series in $l$ variables over $\Omega_{l}$ that converges in an open subset of $\mathbb{C}_{p}^{l}$ of the form $D\left(0, r_{1}\right) \times \cdots \times D\left(0, r_{l}\right)$, with $r_{1}, \ldots, r_{l}>1$, and has the following two properties:

(i) The function from $R$ to $\mathbb{C}_{p}$ defined by $x \mapsto \Theta_{l}(\widehat{x})$ is an additive character $\psi_{l}$ of order $p^{l}$ of $R$, with values in $\Omega_{l}$.

(ii) For each $m \geq 1$, the additive character $\psi_{l, m}$ of $R_{m}$ obtained by composing $\psi_{l}$ with the trace from $R_{m}$ to $R$ can be represented as

$$
\psi_{l, m}(x)=\prod_{i=0}^{m-1} \Theta_{l}\left(F^{i} \widehat{x}\right) .
$$

The aim of this section is to construct a splitting function of level $l$ from the functions $\theta_{i}$ of Section 1. Let

$$
\begin{aligned}
\Theta_{l}: \prod_{i=1}^{l} D\left(0, p^{\left(p^{i-1}(p-1)\right)^{-1}}\right) & \rightarrow \mathbb{C}_{p}, \\
\left(X_{0}, \ldots, X_{l-1}\right) & \mapsto \theta_{l}\left(X_{0}\right) \cdots \theta_{1}\left(X_{l-1}\right) .
\end{aligned}
$$

Then we have

THEOREM 2.3. The function $\Theta_{l}$ is a splitting function of level $l$.

We show this theorem in several steps. Remark first that $\Theta_{l}$ is a power series in $l$ variables over $\Omega_{l}$ that converges in a suitable open subset of $\mathbb{C}_{p}^{l}$ by Proposition 1.5. It remains to show properties (i) and (ii). We begin with an equality of formal power series relating the series $F$ and polynomials arising from addition of Witt vectors; this equality will be the cornerstone of the proof of the theorem. 
LEMMA 2.4. (i) We have the following equality of formal power series in $\mathbb{Z}_{p}[[X, Y]]$ :

$$
\mathrm{AH}(X) \mathrm{AH}(Y)=\prod_{k \geq 0} \mathrm{AH}\left(T_{k}(X, Y)\right)
$$

where, for any $k \geq 0, T_{k}$ is a homogeneous polynomial of degree $p^{k}$ in $\mathbb{Z}[X, Y]$.

(ii) Let $x_{0}, \ldots, x_{l-1}, y_{0}, \ldots, y_{l-1} \in k_{m}$, and let $\widetilde{x}_{0}, \ldots, \widetilde{y}_{l-1} \in \mathcal{T}_{m}$ be their Teichmüller representatives. If we let

$$
\left(z_{0}, \ldots, z_{l-1}\right)=\left(x_{0}, \ldots, x_{l-1}\right) \oplus\left(y_{0}, \ldots, y_{l-1}\right)
$$

in $W_{l}\left(k_{m}\right)$, we have the following congruence in $\mathbb{C}_{p}$ :

$$
\Theta_{l}\left(\widetilde{x}_{0}, \ldots, \widetilde{x}_{l-1}\right) \Theta_{l}\left(\widetilde{y}_{0}, \ldots, \widetilde{y}_{l-1}\right) \equiv \Theta_{l}\left(\widetilde{z}_{0}, \ldots, \widetilde{z}_{l-1}\right)\left[p \gamma_{l}\right] .
$$

Proof. (i) Let $T_{k}(X, Y):=S_{k}(X, 0, \ldots, 0, Y, 0, \ldots, 0)$. Since $S_{k}$ is isobarous of weight $p^{k}$, we see that $T_{k}$ is homogeneous of degree $p^{k}$. Consider, for any $l \geq 0$, the following equality of Witt vectors in $W_{l+1}\left(\mathbb{Z}_{p}[[X, Y]]\right):$

$$
\begin{aligned}
& (X, \ldots, X) \oplus(Y, \ldots, Y)=\bigoplus_{k=0}^{l}\left(V^{k}(X, 0, \ldots, 0) \oplus V^{k}(Y, 0, \ldots, 0)\right) \\
& \quad=\bigoplus_{k=0}^{l} V^{k}\left(T_{0}(X, Y), \ldots, T_{l}(X, Y)\right)=\bigoplus_{k=0}^{l} \bigoplus_{i=0}^{l} V^{k+i}\left(T_{i}(X, Y), 0, \ldots, 0\right) \\
& \quad=\bigoplus_{i=0}^{l} V^{i}\left(\bigoplus_{k=0}^{l} V^{k}\left(T_{i}(X, Y), 0, \ldots, 0\right)\right)=\bigoplus_{i=0}^{l} V^{i}\left(T_{i}(X, Y), \ldots, T_{i}(X, Y)\right) .
\end{aligned}
$$

Now the image under $\Phi_{l}$ of the vector $V^{i}\left(T_{i}(X, Y), \ldots, T_{i}(X, Y)\right)$ in $W_{l+1}\left(\mathbb{Q}_{p}[[X, Y]]\right)$ is

$$
p^{i} T_{i}(X, Y)^{p^{l-i}}+\cdots+p^{l} T_{i}(X, Y)=p^{l} \sum_{j=0}^{l-i} \frac{T_{i}(X, Y)^{p^{j}}}{p^{j}} .
$$

Thus, taking the images under $\Phi_{l}$ (the $l$ th ghost components) of both sides of the above equality of Witt vectors, and dividing by $p^{l}$, we obtain the following congruence in $\mathbb{Q}_{p}[[X, Y]]$ :

$$
G(X)+G(Y) \equiv G\left(T_{0}(X, Y)\right)+\cdots+G\left(T_{l}(X, Y)\right) \bmod (X, Y)^{p^{l+1}} .
$$

Finally, letting $l$ grow to infinity, we get in $\mathbb{Q}_{p}[[X, Y]]$ :

$$
G(X)+G(Y)=\sum_{k \geq 0} G\left(T_{k}(X, Y)\right)
$$

Applying exp to this last equality gives the desired result. 
(ii) We show the congruence by induction on $l$. First note that the equality of formal power series above is valid whenever the two sides converge, that is, for any $x, y \in \mathbb{C}_{p}$ with $|x|,|y|<1$. Let us show the case $l=1$. If $x, y$ are in $k_{m}$, we have

$$
\Theta_{1}(\widetilde{x}) \Theta_{1}(\widetilde{y})=\theta_{1}(\widetilde{x}) \theta_{1}(\widetilde{y})=\operatorname{AH}\left(\gamma_{1} \widetilde{x}\right) \mathrm{AH}\left(\gamma_{1} \widetilde{y}\right)=\prod_{k \geq 0} \mathrm{AH}\left(T_{k}\left(\gamma_{1} \widetilde{x}, \gamma_{1} \widetilde{y}\right)\right) .
$$

Now it is well known that $T_{0}(X, Y)=S_{0}(X, Y)=X+Y$, and we get

$$
\operatorname{AH}\left(T_{0}\left(\gamma_{1} \widetilde{x}, \gamma_{1} \widetilde{y}\right)\right)=\operatorname{AH}\left(\gamma_{1}(\widetilde{x}+\widetilde{y})\right) \equiv \operatorname{AH}\left(\gamma_{1}(\widetilde{x+y})\right)=\Theta_{1}(\widetilde{x+y})\left[p \gamma_{1}\right],
$$

since $\widetilde{x}+\widetilde{y} \equiv \widetilde{x+y}[p]$, and the coefficients of the series $\mathrm{AH}$ are in $\mathbb{Z}_{p}$. On the other hand, if $k \geq 1$, since $T_{k}$ is homogeneous of degree $p^{k}$, we have

$$
\operatorname{AH}\left(T_{k}\left(\gamma_{1} \widetilde{x}, \gamma_{1} \widetilde{y}\right)\right)=\operatorname{AH}\left(\gamma_{1}^{p^{k}} T_{k}(\widetilde{x}, \widetilde{y})\right) \equiv 1\left[p \gamma_{1}\right],
$$

since $v_{p}\left(\gamma_{1}^{p^{k}}\right)=p^{k} /(p-1) \geq 1+1 /(p-1)$, that is, $\gamma_{1}^{p^{k}} \equiv 0\left[p \gamma_{1}\right]$, and $\operatorname{AH}(X) \in 1+X \mathbb{Z}_{p}[[X]]$. Thus we have $\Theta_{1}(\widetilde{x}) \Theta_{1}(\widetilde{y}) \equiv \Theta_{1}(\widetilde{x+y})\left[p \gamma_{1}\right]$, and since both sides of the congruence are $p$ th roots of unity, this proves the case $l=1$ with the help of Corollary 1.2.

Assume we have shown the result for $l-1$. Then

$$
\begin{aligned}
\Theta_{l}\left(\widetilde{x}_{0}, \ldots, \widetilde{x}_{l-1}\right) \Theta_{l}\left(\widetilde{y}_{0}, \ldots, \widetilde{y}_{l-1}\right) & \\
& =\theta_{l}\left(\widetilde{x}_{0}\right) \Theta_{l-1}\left(\widetilde{x}_{1}, \ldots, \widetilde{x}_{l-1}\right) \theta_{l}\left(\widetilde{y}_{0}\right) \Theta_{l-1}\left(\widetilde{y}_{1}, \ldots, \widetilde{y}_{l-1}\right) .
\end{aligned}
$$

From the equality in (i), and since $T_{k}$ is homogeneous of degree $p^{k}$, we have

$$
\theta_{l}\left(\widetilde{x}_{0}\right) \theta_{l}\left(\widetilde{y}_{0}\right)=\operatorname{AH}\left(\gamma_{l} \widetilde{x}_{0}\right) \mathrm{AH}\left(\gamma_{l} \widetilde{y}_{0}\right)=\prod_{k \geq 0} \mathrm{AH}\left(\gamma_{l}^{p^{k}} T_{k}\left(\widetilde{x}_{0}, \widetilde{y}_{0}\right)\right)
$$

and we obtain as above the following congruences (note that from Lemma 1.3, we have $\left.\gamma_{l}^{p^{i}} \equiv \gamma_{l-i}\left[p \gamma_{l}\right]\right)$ :

$$
\begin{aligned}
\operatorname{AH}\left(\gamma_{l}^{p^{i}} T_{i}\left(\widetilde{x}_{0}, \widetilde{y}_{0}\right)\right) & \left.\equiv \operatorname{AH}\left(\gamma_{l-i} \widetilde{T_{i}\left(x_{0}, y_{0}\right.}\right)\right) \\
& =\theta_{l-i}\left(\widehat{\bar{T}_{i}\left(x_{0}, y_{0}\right)}\right)\left[p \gamma_{l}\right] \quad \text { for } i \leq l-1,
\end{aligned}
$$

where $\bar{T}_{i}$ stands for the reduction modulo $p$ of $T_{i}$, and

$$
\operatorname{AH}\left(\gamma_{l}^{p^{i}} T_{i}\left(\widetilde{x}_{0}, \widetilde{y}_{0}\right)\right) \equiv \operatorname{AH}(0)=1\left[p \gamma_{l}\right] \quad \text { for } i \geq l .
$$

Consequently, we obtain

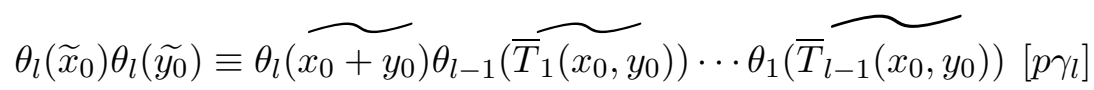

$$
\begin{aligned}
& \left.\equiv \theta_{l}\left(\widetilde{x_{0}+y_{0}}\right) \Theta_{l-1}\left(\widetilde{T_{1}\left(x_{0}, y_{0}\right.}\right), \ldots, \bar{T}_{l-1}\left(x_{0}, y_{0}\right)\right)\left[p \gamma_{l}\right] .
\end{aligned}
$$


Now the result comes from the induction hypothesis, the following equality in $W_{l-1}\left(k_{m}\right)$ :

$$
\left(z_{1}, \ldots, z_{l-1}\right)=\left(\bar{T}_{1}\left(x_{0}, y_{0}\right), \ldots, \bar{T}_{l-1}\left(x_{0}, y_{0}\right)\right) \oplus\left(x_{1}, \ldots, x_{l-1}\right) \oplus\left(y_{1}, \ldots, y_{l-1}\right),
$$

and the fact that $z_{0}=x_{0}+y_{0}$.

We are now ready to prove Theorem 2.3.

Proof of Theorem 2.3. Property (i): From Remark 1.8, and since for $x \in R$ we have $\widehat{x} \in \mathcal{T}^{l}$, it is clear that $\Theta_{l}(\widehat{x})=\Theta_{l}\left(\widetilde{x}_{0}, \ldots, \widetilde{x}_{l-1}\right)$ is a $p^{l}$ th root of unity. Moreover, $\Theta_{l}(\widehat{1})=\Theta_{l}(1,0, \ldots, 0)=\theta_{l}(1)=\zeta_{p l}$ is a primitive $p^{l}$ th root of unity. On the other hand, from Lemma 2.4(ii), for $x, y \in R$ we have

$$
\begin{aligned}
\Theta_{l}(\widehat{x}) \Theta_{l}(\widehat{y}) & =\Theta_{l}\left(\widetilde{x}_{0}, \ldots, \widetilde{x}_{l-1}\right) \Theta_{l}\left(\widetilde{y}_{0}, \ldots, \widetilde{y}_{l-1}\right) \\
& \equiv \Theta_{l}\left(\widetilde{z}_{0}, \ldots, \widetilde{z}_{l-1}\right)\left[p \gamma_{l}\right] \equiv \Theta_{l}(\widehat{x+y})\left[p \gamma_{l}\right]
\end{aligned}
$$

Now since both sides are $p^{l}$ th roots of unity, Corollary 1.2 ensures that the above congruence is in fact an equality. Summing up, we have shown that the map $x \mapsto \Theta_{l}(\widehat{x})$ is an additive character of order $p^{l}$ of $R$, say $\psi_{l}$.

Property (ii): We first show that for $\left(t_{0}, \ldots, t_{l-1}\right)$ in $\mathcal{T}_{m}^{l}$, the product $\prod_{i=0}^{m-1} \Theta_{l}\left(t_{0}^{p^{i}}, \ldots, t_{l-1}^{p^{i}}\right)$ is a $p^{l}$ th root of unity. Actually it is sufficient to show that $\theta_{l}\left(t_{0}\right) \theta_{l}\left(t_{0}^{p}\right) \cdots \theta_{l}\left(t_{0}^{p^{m-1}}\right)$ is a $p^{l}$ th root of unity for any $l$. Since $t_{0}^{p^{m}}=t_{0}$, we have

$$
\begin{aligned}
\left(\theta_{l}\left(t_{0}\right) \theta_{l}\left(t_{0}^{p}\right) \cdots \theta_{l}\left(t_{0}^{p^{m-1}}\right)\right)^{p^{l}} & =\prod_{i=0}^{m-1} \exp \left(p^{l}\left(\gamma_{l} t_{0}^{p^{i}}+\cdots+\frac{\left(\gamma_{l} t_{0}^{p^{i}}\right)^{p^{l}}}{p^{l}}+\cdots\right)\right) \\
& =\exp \left(\left(p^{l} \gamma_{l}+\cdots+\gamma_{l}^{p^{l}}+\cdots\right)\left(t_{0}+\cdots+t_{0}^{p^{m-1}}\right)\right)
\end{aligned}
$$

Note that we can write these equalities since all the terms occurring are in the convergence disk of the exponential (cf. proof of Proposition 1.7); since $\gamma_{l}$ is a zero of $G$, the last term is 1 , and we are done.

From Lemma $2.4\left(\right.$ ii), for any $x_{0}, \ldots, x_{l-1} \in k_{m}$ we have

$$
\prod_{i=0}^{m-1} \Theta_{l}\left(\widetilde{x}_{0}^{p^{i}}, \ldots, \widetilde{x}_{l-1}^{p^{i}}\right) \equiv \Theta_{l}\left(\widetilde{y}_{0}, \ldots, \widetilde{y}_{l-1}\right)\left[p \gamma_{l}\right]
$$

where we have set, in $W_{l}\left(k_{m}\right)$,

$$
\left(y_{0}, \ldots, y_{l-1}\right)=\bigoplus_{i=0}^{m-1}\left(x_{0}^{p^{i}}, \ldots, x_{l-1}^{p^{i}}\right)=\operatorname{Tr}_{W_{l}\left(k_{m}\right) / W_{l}(k)}\left(x_{0}, \ldots, x_{l-1}\right) .
$$

Thus the $y_{i}$ are actually in $k$, and both sides of the congruence are $p^{l}$ th roots of unity; once more, Corollary 1.2 shows that the congruence is an 
equality; finally we get, for any $x \in R_{m}$,

$$
\begin{aligned}
\prod_{i=0}^{m-1} \Theta_{l}\left(F^{i} \widehat{x}\right) & =\prod_{i=0}^{m-1} \Theta_{l}\left(\widetilde{x}_{0}^{p^{i}}, \ldots, \widetilde{x}_{l-1}^{p^{i}}\right)=\Theta_{l}\left(\widetilde{y}_{0}, \ldots, \widetilde{y}_{l-1}\right) \\
& =\psi_{l} \circ w_{m}\left(y_{0}, \ldots, y_{l-1}\right) \\
& =\psi_{l} \circ w_{m}\left(\operatorname{Tr}_{W_{l}\left(k_{m}\right) / W_{l}(k)}\left(x_{0}, \ldots, x_{l-1}\right)\right) \\
& =\psi_{l}\left(\operatorname{Tr}_{R_{m} / R}(x)\right)=\psi_{l, m}(x),
\end{aligned}
$$

and this ends the proof of Theorem 2.3.

3. A Stickelberger theorem for $p$-adic Gauss sums. Let $\zeta_{p^{l}}$ be as above, and $\widetilde{\psi}_{l, m}=\widetilde{\psi}_{l} \circ \operatorname{Tr}_{K_{m} / \mathbb{Q}_{p}}$ be an additive character of order $p^{l}$ of $\mathcal{O}_{m}^{(u)}$, where $\widetilde{\psi}_{l}$ is the additive character of $\mathbb{Z}_{p}$ sending 1 to $\zeta_{p^{l}}$. Let $\zeta$ be a primitive $\left(p^{m}-1\right)$ th root of unity, i.e. a generator of $\mathcal{T}_{m}^{\times}:=\mathcal{T}_{m} \backslash\{0\}$, and $\chi$ be the multiplicative character from $\mathcal{T}_{m}^{\times}$to $\mathbb{C}_{p}^{\times}$, of order $p^{m}-1$, sending $\zeta$ to $\zeta$. For any integer $0 \leq a \leq p^{m}-2$ we define the following Gauss sums:

$$
G_{\mathcal{T}_{m}}\left(\chi^{-a}, \widetilde{\psi}_{l, m}\right)=\sum_{x \in \mathcal{T}_{m}^{*}} \chi^{-a}(x) \widetilde{\psi}_{l, m}(x) .
$$

Note that for $l=1$ these sums coincide with classical Gauss sums over finite fields. They are the same sums as in [8], where they are called incomplete Gauss sums.

These sums lie in the ring of integers $\mathcal{O}_{l, m}$ of $K_{l, m}$, the compositum of $\Omega_{l}$ and $K_{m}$. Notice that $\gamma_{l}$ is a generator of the maximal ideal of $\mathcal{O}_{l, m}$. Our aim is to show

THEOREM 3.1. Let $a$ be an integer such that $0 \leq a \leq p^{m}-2$, and $a=a_{0}+p a_{1}+\cdots+p^{m-1} a_{m-1}$ be its p-adic expansion $\left(0 \leq a_{i} \leq p-1\right)$. Set $s(a):=a_{0}+a_{1}+\cdots+a_{m-1}$ and $p(a):=a_{0} ! \cdots a_{m-1}$ !. Then we have the following congruence in $\mathcal{O}_{l, m}$ :

$$
G_{\mathcal{T}_{m}}\left(\chi^{-a}, \widetilde{\psi}_{l, m}\right) \equiv-\frac{\gamma_{l}^{s(a)}}{p(a)}\left[\gamma_{l}^{s(a)+1}\right] .
$$

REMARK 3.2. Note that by the Remark 1.8(ii) we can replace $\gamma_{l}$ by $\zeta_{p^{l}}-1$ in the preceding congruence.

Proof of Theorem 3.1. We first rewrite the Gauss sum. The character $\widetilde{\psi}_{l, m}$ factors to the character $\psi_{l, m}: \mathcal{O}_{m}^{(u)} / p^{l} \mathcal{O}_{m}^{(u)}=R_{m} \rightarrow \mathbb{C}_{p}^{*}$, obtained by composing $\psi_{l}: R \rightarrow \mathbb{C}_{p}$, the character sending 1 to $\zeta_{p^{l}}$, with the trace from $R_{m}$ to $R$. Since an element $x$ of $\mathcal{T}_{m}$ is sent via $w_{m}^{-1}$ to the element $(\bar{x}, 0, \ldots, 0)$ of $W_{l}\left(k_{m}\right)$, we get

$$
\widetilde{\psi}_{l, m}(x)=\Theta_{l}(x, 0, \ldots, 0) \cdots \Theta_{l}\left(x^{p^{m-1}}, 0, \ldots, 0\right)=\theta_{l}(x) \cdots \theta_{l}\left(x^{p^{m-1}}\right) ;
$$


thus we can write, in $\mathbb{C}_{p}$ (actually in $\mathcal{O}_{l, m}$ ),

$$
\begin{aligned}
G_{\mathcal{T}_{m}}\left(\chi^{-a}, \tilde{\psi}_{l, m}\right) & =\sum_{x \in \mathcal{T}_{m}^{*}} x^{-a_{0}-p a_{1}-\cdots-p^{m-1} a_{m-1}} \theta_{l}(x) \cdots \theta_{l}\left(x^{p^{m-1}}\right) \\
& =\sum_{x \in \mathcal{T}_{m}^{*}}\left(\sum_{n \geq 0} \lambda_{n, l} x^{n-a_{0}}\right) \cdots\left(\sum_{n \geq 0} \lambda_{n, l}\left(x^{p^{m-1}}\right)^{n-a_{m-1}}\right) \\
& =\sum_{n_{0}, \ldots, n_{m-1} \geq 0} \lambda_{n_{0}, l} \cdots \lambda_{n_{m-1}, l} \sum_{x \in \mathcal{T}_{m}^{*}} x^{n_{0}-a_{0}+\cdots+p^{m-1}\left(n_{m-1}-a_{m-1}\right)} \\
& =\sum_{n_{0}, \ldots, n_{m-1} \geq 0} \lambda_{n_{0}, l} \cdots \lambda_{n_{m-1}, l} \sum_{x \in \mathcal{T}_{m}^{*}} x^{n_{0}+\cdots+p^{m-1} n_{m-1}-a} \\
& =\left(p^{m}-1\right) \quad \sum_{\substack{n_{0}, \ldots, n_{m-1} \geq 0 \\
n_{0}+\cdots+p^{m-1} n_{m-1} \equiv a\left[p^{m}-1\right]}} \lambda_{n_{0}, l} \cdots \lambda_{n_{m-1}, l}
\end{aligned}
$$

(notice that the sum $\sum_{x \in \mathcal{T}_{m}^{*}} x^{n}$ is $p^{m}-1$ if $n \equiv 0\left[p^{m}-1\right]$ and zero otherwise). Now $v_{p}\left(\lambda_{n_{0}, l} \cdots \lambda_{n_{m-1}, l}\right) \geq\left(n_{0}+\cdots+n_{m-1}\right) /\left(p^{l-1}(p-1)\right)$, and

$$
\lambda_{a_{0}, l} \cdots \lambda_{a_{m-1}, l}=\frac{\gamma_{l}^{s(a)}}{p(a)}
$$

by the description of the coefficients $\lambda_{n, l}$ for $n \leq p-1$ in Proposition 1.5; thus the theorem comes from the following lemma.

LEMMA 3.3. Let $0 \leq a \leq p^{m}-2$ and $n_{0}, \ldots, n_{m-1}$ be $m+1$ nonnegative integers such that

$$
n_{0}+\cdots+p^{m-1} n_{m-1} \equiv a\left[p^{m}-1\right] .
$$

If $a=a_{0}+p a_{1}+\cdots+p^{m-1} a_{m-1}$ is the $p$-adic expansion of the integer $a$, then

$$
n_{0}+\cdots+n_{m-1} \geq s(a)=a_{0}+\cdots+a_{m-1},
$$

and equality occurs if and only if $n_{0}=a_{0}, \ldots, n_{m-1}=a_{m-1}$.

Proof. Set $n_{0}+\cdots+p^{m-1} n_{m-1}=a_{0}+p a_{1}+\cdots+p^{m-1} a_{m-1}+k\left(p^{m}-1\right)$. We must have $k \geq 0$ since $0 \leq a \leq p^{m}-2$. We rewrite this as

$$
\begin{aligned}
& n_{0}+\cdots+p^{m-1} n_{m-1} \\
& \quad=a_{0}+k(p-1)+p\left(a_{1}+k(p-1)\right)+\cdots+p^{m-1}\left(a_{m-1}+k(p-1)\right) .
\end{aligned}
$$

Reducing this last equality modulo $p$, we get $n_{0} \equiv a_{0}-k[p]$, and there exists an integer $k_{1}$ such that $n_{0}=a_{0}-k+k_{1} p$. Moreover $k_{1}$ is a nonnegative integer since $0 \leq a_{0} \leq p-1$, and $k, n_{0} \geq 0$. We can rewrite the first equality of this proof as $p\left(n_{1}+k_{1}\right)+\cdots+p^{m-1} n_{m-1}=p\left(a_{1}+k p\right)+\cdots+p^{m-1}\left(a_{m-1}+k\left(p^{m}-1\right)\right)$. 
Dividing both sides by $p$ and reducing modulo $p$ yields $n_{1}+k_{1}=a_{1}+p k_{2}$ with $k_{2}$ an integer, nonnegative since $k_{1} \geq 0$ and $0 \leq a_{1} \leq p-1$. We can repeat this process to get nonnegative integers $k_{3}, \ldots, k_{m-1}$ such that $n_{i}+k_{i}=a_{i}+p k_{i+1}$ for $1 \leq i \leq m-2$, and $n_{m-1}+k_{m-1}=a_{m-1}+k p$. Summing all these equalities, we get

$$
\begin{gathered}
n_{0}+\cdots+n_{m-1}+k_{1}+\cdots+k_{m-1}=a_{0}-k+k_{1} p+a_{1}+k_{2} p+\cdots+a_{m-1}+k p, \\
n_{0}+\cdots+n_{m-1}=a_{0}+a_{1}+\cdots+a_{m-1}+\left(k+k_{1}+\cdots+k_{m-1}\right)(p-1) .
\end{gathered}
$$

Since $k$ and the $k_{i}$ are nonnegative integers, this last equality proves the lemma.

REMARK. Note that for $l=1$, we obtain the classical Stickelberger theorem (cf. [2], [7]).

\section{References}

[1] Y. Amice, Les nombres p-adiques, Presses Univ. France, Paris, 1975.

[2] B. C. Berndt, R. J. Evans and K. S. Williams, Gauss and Jacobi Sums, Wiley, New York, 1998.

[3] N. Bourbaki, Algèbre Commutative IX, Hermann, Paris, 1962.

[4] B. M. Dwork, On the zeta function of an hypersurface, Inst. Hautes Études Sci. Publ. Math. 12 (1962), 5-68.

[5] N. Koblitz, p-adic Numbers, p-adic Analysis and Zeta-Functions, Grad. Texts in Math. 58, Springer, New York, 1977.

[6] P. V. Kumar, T. Helleseth and A. R. Calderbank, An upper bound for Weil exponential sums over Galois rings and applications, IEEE Trans. Inform. Theory 41 (1995), 456-468.

[7] S. Lang, Algebraic Number Theory, Grad. Texts in Math. 110, Springer, 1994.

[8] P. Langevin and P. Solé, Gauss sums over quasi-Frobenius rings, in: Finite Fields and Applications, Springer, 2001, 329-340.

[9] B. R. MacDonald, Finite Rings with Identity, Dekker, 1974.

[10] J.-P. Serre, Corps locaux, Hermann, Paris, 1963.

[11] A. G. Shanbag, P. V. Kumar and T. Helleseth, An upper bound for a hybrid sum over Galois rings with applications to aperiodic correlation for some q-ary sequences, IEEE Trans. Inform. Theory 42 (1996), 250-254.

Équipe "Algèbre Arithmétique et Applications"

Université Antilles Guyane

Campus de Fouillole

97159 Pointe à Pitre Cedex - FWI

E-mail: rblache@univ-ag.fr 